\title{
Adaptive Description of ATM Traffic Flows
}

\author{
Juergen Rochol \\ Mouriac Halen Diemer \\ (juergen@inf.ufrgs.br) \\ (mouriac@univates.br) \\ Universidade Federal do Rio Grande do Sul - UFRGS \\ Instituto de Informática - Departamento de Informática Aplicada \\ CEP 91501-970 Av. Bento Gonçalves, 9500 Porto Alegre - RS \\ Caixa Postal 15064
}

\begin{abstract}
Today, the great challenge in broad band networks, such as ATM, is to support applications that need guaranteed QoS. Efficient resource allocation for connections that need guaranteed QoS is only possible if the application can provide a precise and reliable traffic descriptor that the network can understand. This work presents a methodology to obtain the parameters of the traffic descriptor of an ATM application from an initial sample of the flow. Based on real-time measurements of the flow, we suggest periodic renegotiations of the parameters, every time they vary above or below their predefined limits. We apply this method to a VBR MPEG video flow and demonstrate its performance. Observed results are discussed and some future uses of the methodology are suggested.
\end{abstract}

Keywords: ATM, traffic descriptor, traffic shaping, traffic policing.

\section{INTRODUCTION}

Modern broad band application typically require variable band width and specific performance requirements. ATM technology allows the implementation of a network capable of supporting the QoS requirements of such applications, as long as the application provides the network with a flow of cells within negotiated UPC (user parameter control) parameters. This set of UPC parameters is part of the application's traffic descriptor, which must be able to capture the main statistical characteristics of the flow. This is the only way a network can allocate the required resources to meet such application's QoS requirements.

However, the application often has no means to provide precise information about its flow behavior, which forces the network to adopt QoS standards based solely on the application's service category, which not always translates as an efficient allocation of network resources concerning band and buffer space. In such case, a feasible alternative being proposed would be to try to use an initial sample of the flow to infer a set of attempt-parameters and renegotiate them as significant deviations of these parameters from the current flow occur.

Figure 1 shows a block diagram of the traffic model we are proposing and its main function blocks. The description and control block acquires UPC parameters from an initial sample of the flow, which are then sent to the network by the signaling block through signaling protocol Q.2963.2 of ITU-T. The shaping block monitors UPC parameters behavior statistics and starts their renegotiation when they vary above or below predefined values.

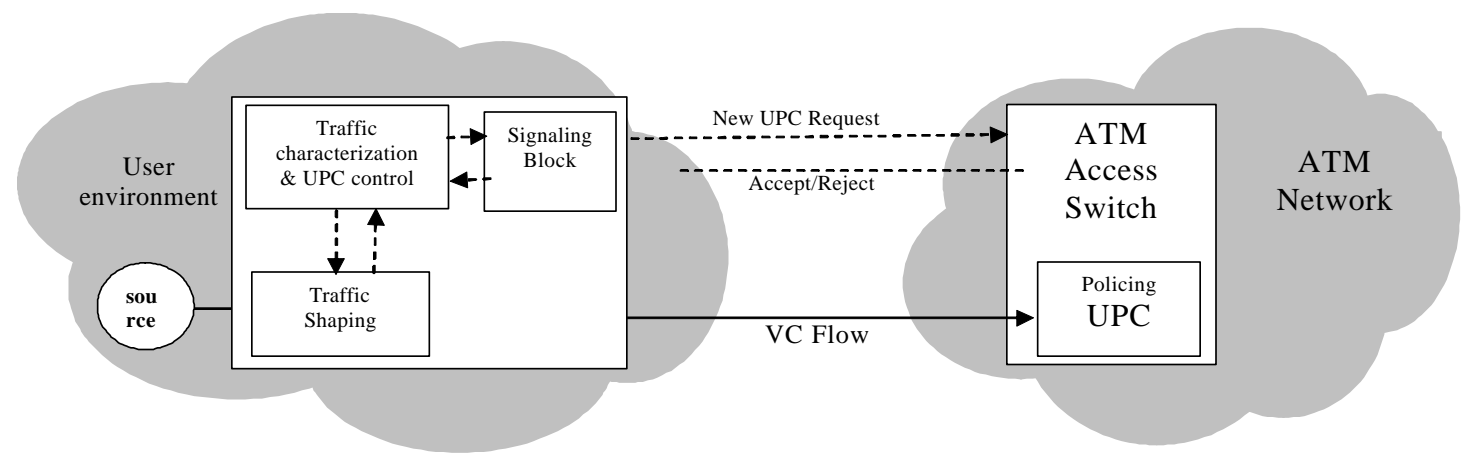

Fig. 1. Model for an adaptive traffic description and traffic shaping module for a VC flow in ATM

\footnotetext{
${ }^{1}$ This work was partially financed by FAPERGS - Fundação de Amparo à Pesquisa do Rio Grande do Sul , Processo n. 01/0575.9
} 
This work is organized as follows: section 2 defines a model for the adaptive shaping of a flow associated to a generic VBR virtual connection (VC) based on UPC parameters obtained from the description module. We outline the theoretical foundations of the method adopted to obtain UPC parameters and present a simplified, practical method, to obtain UPC parameters from a real flow sample. Section 3 presents simulations and experimental results obtained when the method was applied to an MPEG video flow. in section 4 we present a discussion of the results obtained and also some conclusions and prognoses, forecasting the use of the method in some specific areas, besides possible extensions to it.

\section{A. Adaptative Shaping of an ATM Flow}

The strategy that the ATM Forum adopted to describe cell flow is based on a description on two time scales: (1.) describing the behavior of short-term bursts, by using parameters such as peak rate (PCR) and its corresponding jitter rate (CDVT), and (2.) describing the flow's long-term behavior, or the flow's statistical description, in which the statistical time interval corresponds to the connection time. The parameters used in this case are the statistical cell rate (or sustainable cell rate, SCR) and a tolerance regarding such rate, which is, however, bound to the peak rate, and called burst tolerance (BT). CDVT is not exactly a source-generated traffic-related parameter, but a network-engineering parameter, since it accounts for the jitter entering the flow as it travels from the source to entry in the network through the UNI interface. Burst tolerance BT is related to the maximum burst size (MBS) and the average rate (SCR) and peak rate (PCR) through expression (1) [2].

$$
B T=\tau_{s}=(M B S-1)\left(\frac{1}{S C R}-\frac{1}{P C R}\right)
$$

Characterizing, or describing, variable bit rate (VBR) flows is a generic strategy, since one can consider any flows in other ATM Forum service categories to be particular cases of this generic VBR flow.

In order to police flows, ATM Forum [2] has suggested two equivalent versions of leaky buckets (LB), known as GCRA (Generic Cell Rate Algorithm). They are the continuous-state leaky bucket and the virtual scaling leaky bucket. A more detailed analysis of their operation and performance can be found in [3].

Leaky bucket (LB) is a simple count mechanism that can be used for shaping and policing traffic flows. Our analysis uses the leaky bucket model suggested by Mark [4]. In this model, leaky bucket operation is described by a fictitious $. / D / 1 / B$ queue system served at a constant $\mu$ rate and having buffer capacity $B$, described as a leaky bucket $L B(\mu, B)$, as shown in Figure 2a.

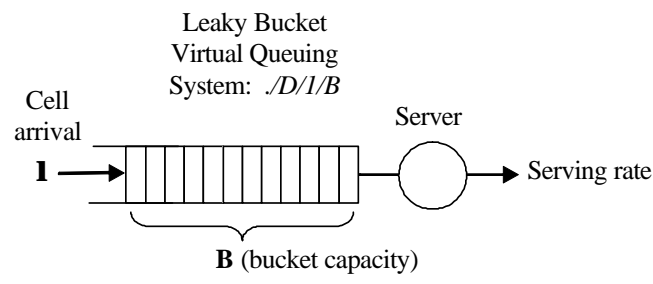

(a)

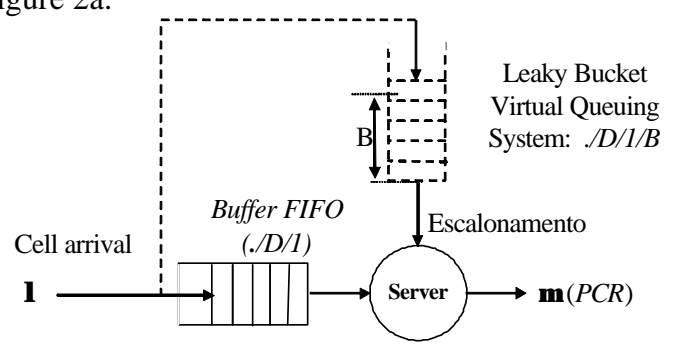

(b)

Fig. 2. (a) Model for a Leaky Bucket $L B(\mu, B)$ and (b) Model for a Leaky Bucket Shaper (LBS) for PCR conformance.

A leaky bucket, when used to conform, or shape traffic, is also parametered through $(\mu, B)$ and operates similarly to a policing leaky bucket, except that non-conforming cells are not marked or discarded, but rather delayed. The operation can be understood in terms of a $. / D / 1$ queue system of infinite capacity, as shown in Figure 2b. If, upon the arrival of a cell, the number of clients on the fictitious queue is $B$ or more, this cell is put on the infinite capacity buffer served on a FIFO basis. Every time a client leaves the fictitious queue, a cell is sent from the FIFO buffer. This model is equivalent to the ones known as Token Bucket, or leaky bucket with a memory or buffer [5], [6].

The Double Virtual Scaling Shaper (DVSS) on our approach uses two GCRA virtual scaling algorithms - one for PCR and another for SCR. DVSS operation is based on the simultaneous supervision of two parameters: conformed emission time at the cell's peak rate (CETP), and conformed emission time at the cell's sustainable rate (CETS). Based on these parameters, the cell's conformed emission time (CET) can be defined to ensure simultaneous conformity of the flow to CETp and CETs as follows: 


\section{$\mathrm{CET}=\max (\mathrm{CET}-\mathrm{BT}, \mathrm{CETp})$}

The operation can be described as follows: upon the actual arrival ta time of a cell, its CET is determined. If CET $\leq t a$, it is scaled; otherwise it stays on buffer for later transmission. Figure 3 illustrates the operation of the algorithm.

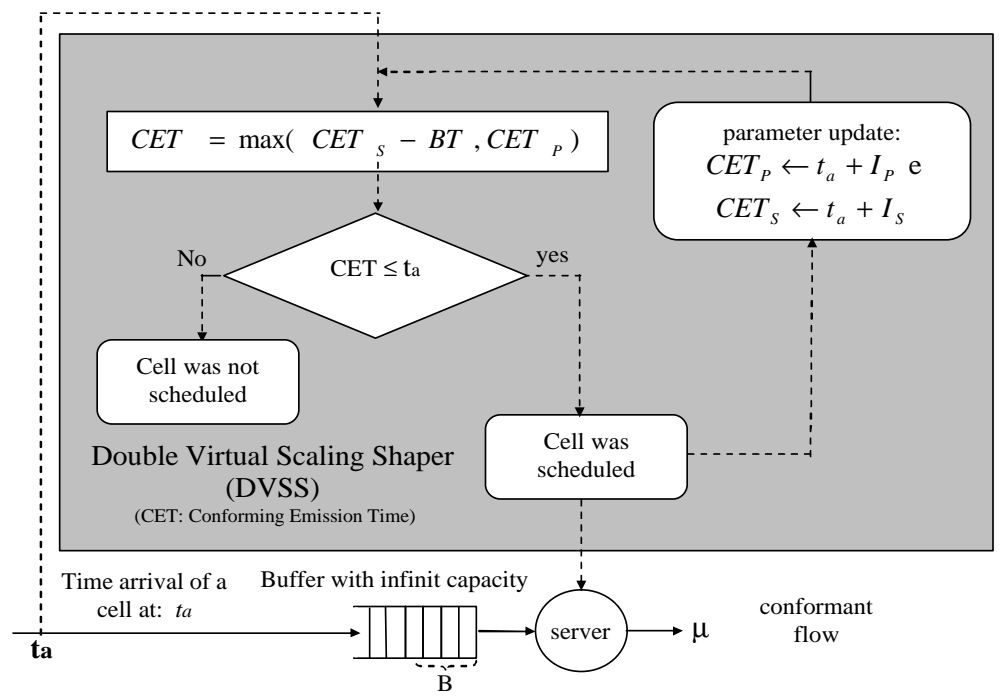

Fig. 3. Double Virtual Scaling Shaper (DVSS)

The shaper ensures that the peak rate does not exceed $\lambda_{p}$, the long-term average rate does not exceed $\lambda s$ and the maximum burst length (transmitted at peak rate) does not exceed:

$$
B_{c}=B_{s} \frac{\lambda_{p}}{\left(\lambda_{p}-\lambda_{s}\right)}
$$

The value of $B c$, in such case, can be easily related to the MBS parameter of the connection traffic descriptor defined in (1). Conformance can be adapted in relation to the statistical variations of this flow, by observing its behavior at predefined time intervals and by regularly renegotiating the new sample-inferred parameters with the network.

\section{B. Approximate Statistical Description}

Here follows an extension of the methodology of Mark et al. [4] that allows its direct application on samples of generic VBR flows to obtain statistical parameters that are then mapped into UPC parameters in order to shape this flow.

The process of approximate statistical description, $\hat{C}_{s}$, is based on the behavior of a pool of leaky bucket shapers, each one of them described by traffic parameters that have been estimated from an initial flow sample. Assuming that the pool of shapers is modeled according to a queue system set and that the cell flow of the sample one wishes to describe is applied to all shapers of this pool. Moreover, each shaper has a separate and constant service rate equal to $\mu i$. The values of $\mu i$ must be in the range $\lambda m<\mu i<\lambda p$, where $\lambda p$ and $\lambda m$ are, respectively, the peak rate and the average rate of the flow sample. In practical terms, the peak rate and average rate values can be obtained from a direct real-time assessment of the flow sample. Besides that, assuming that $W$ represents the average waiting time of any given cell in this queue system, when the latter is balanced, using the function of probability distribution of the complementary waiting time as its approximate exponential form [7], the probability that the waiting time of a buffered cell be greater than a given time $t$ is expressed as:

$$
P(W>t) \approx a(\mu) e^{-b(\mu) t}
$$

In that expression, $a$ and $b$ depend on $\mu$. If $t$ is measured in seconds, then the dimension of $b$ is an inverse of seconds. Given relatively weak hypotheses of the arrival process nature, exponential form (3) has an asymptotic curve for very large $t$, i.e., $e^{b t} P(W>t) \rightarrow a \quad$ (for $t \rightarrow \infty$ ). Mark and Ramamurthy [4] show that 
this particular asymptotic curve is true for a rather general class of arrival processes with Markovian structure. They also show that the weaker asymptotic form $-t^{-1} \log P(W>t) \rightarrow-b \quad$ (for $t \rightarrow \infty$ ) -, is valid under much more generic conditions, i.e., it dispenses with the Markovian supposition of distribution of cell arrival. However, approximation (3) is exact in the case of ON/OFF Markovian flow sources. For relatively small to moderate size buffers, one obtains sufficiently precise results for a great variety of classes of Markovian models, including self-similar traffic models [8].

Parameters $a(\mu)$ and $b(\mu)$ offer a statistical description of the flow as they describe the effect of the cell flow when applied to a queue with constant service rate $\mu$. A more complete description of the cell flow can be obtained by relating $a(\mu)$ and $b(\mu)$ with $\mu$ values in interval $(\lambda m, \lambda p)$ as follows:

$$
\hat{C}_{s}=\left[\lambda p ; \lambda m ;(a(\mu), b(\mu)) \text { where } \lambda_{m}<\mu<\lambda_{p,}\right]
$$

Values for $a(\mu)$ and $b(\mu)$ can be chosen in a way that approximation (3) is true for $t$ values within the interval of interest. Assuming that expression (3) is an equality, with basis on [10] one can say that:

$$
\frac{a(\mu)}{b(\mu)}=E[W]=\tau_{r}(\mu) E\left[S_{a}\right]+\frac{E\left[Q_{a}\right]}{\mu},
$$

Where $S a$ represents the number of service cells $\left(S_{a} \in\{0,1\}\right.$ ), $Q a$ represents the number of queued cells and $\tau r(\mu)$ represents the average service time left for the service cell (as long as there is a servicing cell). From expression (3), $a(\mu)$ can be calculated assuming that:

$$
P(W>0)=a(\mu)=E[S a]
$$

By defining $E[Q a]=q(\mu)$ and solving for $b(\mu)$, one has:

$$
b(\mu)=\frac{a \mu}{a \mu \tau_{r}+q}
$$

In order to complete UPC parametrization of the flow, it is also necessary to obtain the parameters of minimum capacity $\mathrm{B}$ of the leaky bucket as a function of the flowing rate $\mu$, where $\lambda m<\mu<\lambda p$. Flowing rate $\mu$ of the bucket can be defined as a function of a given conformance probability $\varepsilon$ or, when more convenient, as a function of a given probability of delay the flow can tolerate.

In [10], it is shown that the minimum size of the bucket in such case can be calculated as:

$$
B\left(\hat{C}_{s}, \mu, \varepsilon\right)=\frac{\mu}{b(\mu)} \log \left(\frac{a(\mu)}{\varepsilon}\right)
$$

Instead of obtaining the minimum bucket capacity as a function of a conformance probability, capacity B can be dimensioned according to delay criterion $\bar{D}$. If the application tolerates some degree of delay in relation to its flow, it may be more convenient to specify the limit of flow conformance as a function of this delay. Once again, [10] shows that in such case the minimum size of the bucket can be calculated as:

$$
B\left(\hat{C}_{s}, \mu, \bar{D}\right)=\frac{\mu}{b(\mu)} \log \left(\frac{a(\mu)}{b(\mu) \bar{D}}\right)+1
$$

\section{A PRACTICAL METHOD TO OBTAIN UPC PARAMETERS}

What follows is a practical way for real-time estimation the UPC parameters of a flow, from an empirical methodology suggested in [4]. Parameter estimation is based on observing a sample of this flow for a time interval $\mathrm{T}$ and the method follows five phases:

a. Estimating average and peak rates from a flow sample.

b. Choosing a set of values that may be the sustainable rate.

c. Obtaining the approximate statistical description of the flow $\left(\hat{C}_{s}\right)$.

d. Calculating a minimum bucket size for each possible value, enough to meet the demands of delay or user shaping probability.

e. Obtaining sustainable rate $\lambda s$ and bucket capacity $\mathrm{Bs}$, and mapping UPC traffic parameters of the flow. 


\section{a. Estimating average and peak rates from a flow sample}

Considering the estimated average rate $\hat{\lambda}_{m}$ and the estimated peak rate $\hat{\lambda}_{p}$ on a sampling window $T$, the typical value of $T$ for video services, according to Knightly [11], can be from two to four seconds. A given interval $T$ is subdivided into $M$ subintervals of fixed length $t$, so that $t=T / M$. In case of ON/OFF traffic, for instance, $t$ can be the sum of the ON interval and the OFF interval $(t=t o n+t o f f)$. As for MPEG traffic, one can use $t$ as the duration of each frame, i.e., $t=33 \mathrm{~ms}$ and thus $M \approx 100$. Supposing that a total $A$ cells are issued during a $T$ time interval, the estimated average rate will then be $\hat{\lambda}_{m}=A / T$. The peak rate, on its turn, when unknown beforehand, can be estimated from $\hat{\lambda}_{p}=\max \{n i / t\}$, where $1 \leq i \leq M$, ni corresponding to the number of cells emitted on the $i$-th $t$ time interval.

\section{b. Choosing a set of values that may be the sustainable rate}

A set of possible $\mu$ values that may be the estimated sustainable rate $\hat{\lambda}_{s}$, equally spaced on interval $(\lambda m, \lambda p)$, written as: $\mu 1, \mu 2, \ldots \mu N$, where the number $N$ of possible values must be a commitment between how close one wishes to get to $\hat{\lambda}_{s}$ and the necessary processing time to obtain it. Assuming that time is normalized in relation to the time of cell transmission through the link level, one must choose the increment between two subsequent rate values as an integer, i.e., $\Delta\left\lfloor\mu_{i+1}\right\rfloor-\left\lfloor\mu_{i}\right\rfloor=\Delta=1,2,3, \ldots$

\section{c. Obtaining the approximate statistical description of the flow}

For the estimated statistical description $\hat{C}_{s}$, it is considered that, for each of the $N$ possible rates $\mu i(i$ $=1,2, \ldots N)$, a leaky bucket $X i$ - which is incremented by one for each arriving cell and decremented at a constant rate $\mu i$. For each rate $\mu i$, the estimated values of parameters $\hat{a}\left(\mu_{i}\right)$ and $\hat{b}\left(\mu_{i}\right)$ are obtained as follows: each fictitious queue is sampled at the moment an arbitrary cell arrives, sampling is done by skipping a random $X j$ number of cells between moment $j-1$ and moment $j$ of sampled arrivals. Since all $N$ queues are shown at the same time, only one random number is needed for all queues at the moment of sampling, thus reducing the processing time of the algorithm.

During interval $T, M$ samplings are collected on the $N$ queues. On any given $j$ sampling, the following queue values will be observed:

$S j$ : number of clients attended $(S j \ni\{0,1\})$

$Q j$ : number of clients on queue

$T j$ : service time left for the client being attended (if this is the case)

One can then obtain the following approximate mean values:

$$
\hat{a}=\frac{1}{M} \sum_{j=1}^{M} S_{j}, \quad \hat{q}=\frac{1}{M} \sum_{j=1}^{M} Q_{j} \text { and } \hat{\tau}_{r}=\frac{1}{\hat{a} . M} \sum_{j=1}^{M} T_{j} \approx \frac{1}{2 \mu}
$$

Assuming that the average attending time of a cell in service can be neared to $\tau_{r} \approx 1 / 2 \mu$ and the value of $b$ can be obtained by expression (7):

$$
\hat{b}=\frac{\hat{a} \cdot \mu}{\mu \cdot \hat{\tau}_{r} \cdot \hat{a}+\hat{q}} \approx \frac{\hat{a} \mu}{\hat{a} / 2+\hat{q}}
$$

The statistical description is as follows:

$$
\hat{C}_{s}=\left[\hat{\lambda}_{p} ; \hat{\lambda}_{m} ;\left(\hat{a}\left(\mu_{i}\right), \hat{b}\left(\mu_{i}\right)\right), i=1, \ldots, N\right]
$$

\section{d. Determining bucket capacity}

Once the approximate statistical description $\hat{C}_{s}$ of the flow has been obtained, one can determine the capacity of bucket $B s\left(\hat{C}_{s}, \mu, \varepsilon\right)$ for each $\mu$ rate as a function of a conformance probability $\varepsilon$ according to relation (8). If the flow allows for a little large conformance delay, the bucket minimum capacity can be determined as a function of a given delay limit $\bar{D}$ intended, through expression (9).

e. Obtaining the sustainable rate

Mapping UPC parameters of the flow from the approximate statistical description $\hat{C}_{s}$ can be done in two ways: by imposing a delay limitation, or by imposing a conformance probability. The current analysis used 
the latter because it is more practical concerning traffic flows. Thus, the set of traffic descriptors acceptable for the flow can be written as:

$$
D_{T}:\left[\hat{\lambda}_{p}, \mu_{i}, B_{s}\left(\hat{C}_{s}, \mu_{i}, \bar{D}\right] \text { where } i=1, \ldots, N\right.
$$

In order to choose the most adequate traffic descriptor, the option is for the one with the smallest effective band associated. To do so, an expression adapted from [9] was used, which expresses the effective band of a flow as a function of the parameters of its traffic descriptor. For an ON/OFF flow, the effective band can be expressed as:

$$
b_{e f f}\left(\lambda_{s}, \lambda_{p}, B_{s}\right)=\lambda_{s}+K \frac{\lambda_{s}\left(\lambda_{p}-\lambda_{s}\right) B_{s}}{\lambda_{p}}
$$

The effective band associated to a UPC-controlled flow equals rate $\lambda s$ plus a term that takes into account the burstness associated to such flow. The value of constant $K$, in this expression, depends on the characteristics of the ATM multiplexer and the burstness of the flow and can be determined, according to [4], by the expression:

$$
K=\frac{\log \left(1 / \varepsilon_{\text {mux }}\right)}{2 k B_{\text {mux }}}
$$

In this expression, Emux is the probability of loss of MUX cells, $k$ is a coefficient related to the burstness of the flow and Bmux is the capacity of the MUX buffer. According to experiment results in [4], the value of $K$ for CBR-type flows is near zero, whereas for ON/OFF flows, which can be rather explosive, it can be near one. It was considered that $K$ had an intermediary value $(K \approx 0,01)$, aiming at keeping the generality intended for the method. Parameter $i$ is chosen by minimizing the following expression of effective band associated to flow:

$$
b_{\text {eff min }}=\min \left(\lambda_{p}, \mu_{i}+\frac{0.001 \mu_{i}\left(\lambda_{p}-\mu_{i}\right) B_{s}}{\lambda_{p}}\right)(1 \leq i \leq N)
$$

The value of $\hat{\imath}$ within the $N$ possible values of $i$ was called minimum effective band, which minimizes the previous expression. The parameters of the traffic descriptor $\left(\lambda_{s}, \lambda_{p}, B_{s}\right)$, associated to the considered flow, can then be approximated as:

$$
\lambda_{p}=\hat{\lambda}_{p} \quad \lambda_{s}=\mu_{\hat{i}} \text { e } B_{s}=B_{s}\left(\hat{C}_{s}, \lambda_{s}, \bar{D}\right)
$$

It can be noticed that if the estimated values of the peak and average rates are too close, i.e., CBR flows, then it is true that:

$$
\lambda_{p}=\hat{\lambda}_{p}, \lambda_{s}=\hat{\lambda}_{p} \text { e } B_{s}=1
$$

\section{SIMULATION AND EXPERIMENTAL RESULTS}

In order to assess the performance of the practical method in obtaining UPC parameters, an application was sought that were as close as possible to real-time processing, which means that the UPC parameters must be inferred from a small initial sample (duration of a few seconds) because such time may be the fixed delay to be introduced on the flow.

The practical method was applied to a flow of real video compressed according to the MPEG standard [12]. Under this standard, the video is transmitted according to a sequence of frames of varied sizes. When transmitting such flow on an ATM network, each frame is segmented into cells. Cells are transmitted at a constant rate $\lambda p$ at the beginning of a frame interval until the frame has been transmitted. The source then silences until the beginning of a new frame interval, which results in a VBR ON/OFF flow with alternate ON and OFF periods. On MPEG coding, there are three types of frames: frames I (Intracoded frames), frames P (Predicted-coded frames) and frames B (Bidirectional-coded frames). Frames I follow codification called intraframe and contain the complete information of a scene, whereas frames $\mathrm{P}$ and $\mathrm{B}$, or interframes, codify only a prediction of movement based on other frames. In general, the size of these frames have the relation $I>$ $P>B$. The repetition sequence of different frames is fixed and periodical, determined through Group of Pictures (GOP). The GOP sequence is characterized by two parameters, $N$ and $M$, which define the distance between $I$ frames and $P$ frames, respectively.

Our evaluation used the first 1,200 frames of the first scenes of MPEG video "Star Wars", which 
presents a fixed frames rate of $30 \mathrm{fps}$, which corresponds to a frame interval of $33 \mathrm{~ms}$. Figure 4 shows a graph of the variation of the size of frames in this sequence, whose approximate duration is 40s. This sequence was divided into 10 segments, called samples, each with 120 frames and approximate duration of $4 \mathrm{~s}$. Sample-1 is the name given to the frames corresponding to the first $4 \mathrm{~s}$, sample- 2 are the subsequent four, and so on. Figure 5 shows the graph of the 120 frames that make up sample- 1 .

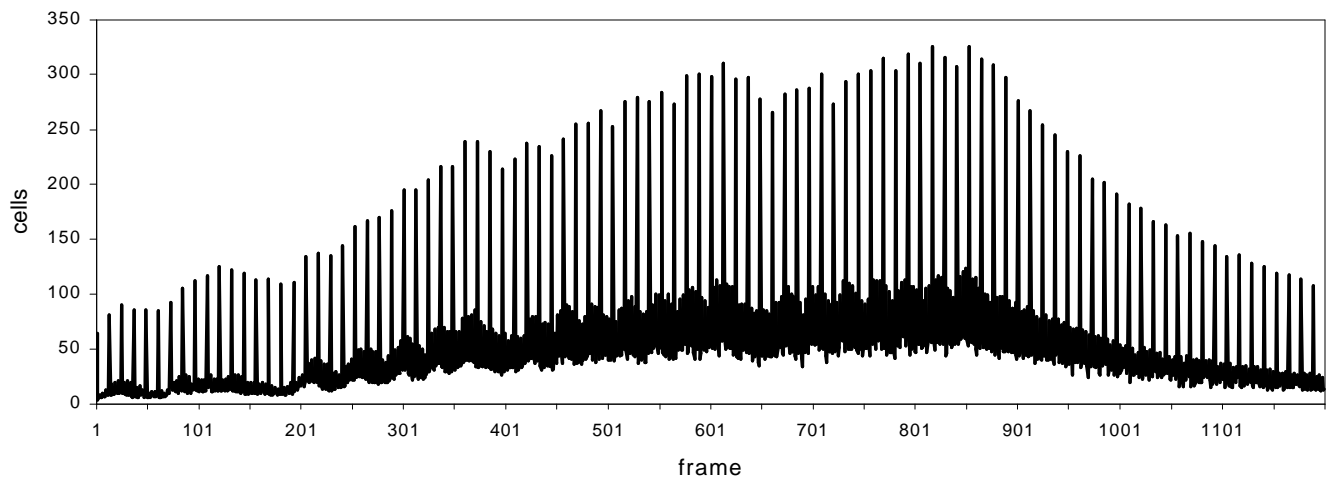

Fig. 4. Size of frames of the first 40s of Star Wars video.

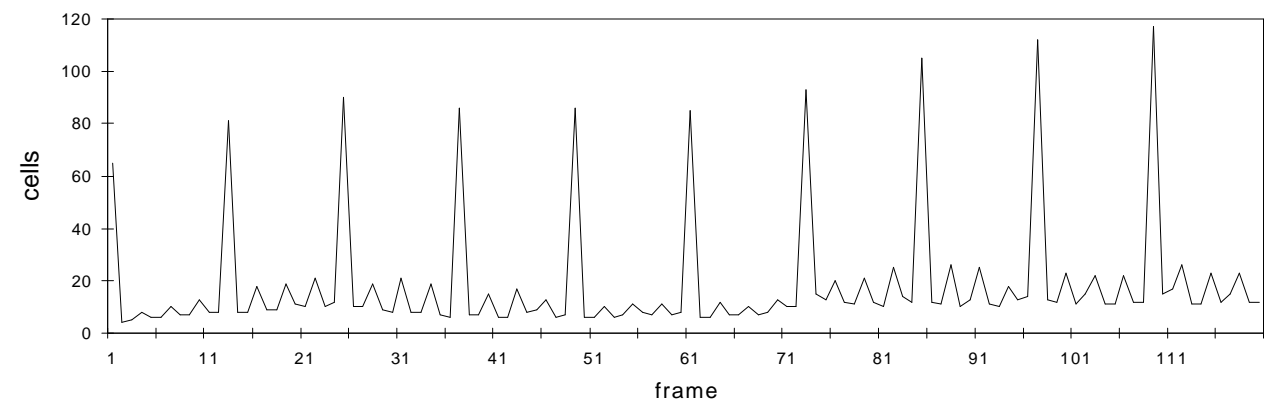

Fig. 5. Size of frames of sample-1 of Star Wars MPEG video.

The first 120 frames of the Star Wars sequence (sample-1) present a frame with maximum size of 117 cells, a frame with minimum size of 4 cells and an average size of 18.45 cells/frame. Assuming a link access of 154.18 Mbit/s, the cell insertion time (or timeslot-ts) is $2.75 \mu \mathrm{s}$. For simplicity reasons, time in our analysis has been normalized in relation to this unit. For sample-1, the peak rate was estimated as $\lambda p=9,7510^{\wedge}-3$, and the average rate as $\lambda \mathrm{m}=1,5310^{\wedge}-3$, in cell units per time-slot. For further simplification, the inverse of the rates was considered, i.e., a minimum interval between cells of $t p=1 / \lambda p \approx 103$ ts and an average interval between cells of $t m=1 / \lambda m \approx 648 \mathrm{ts}$. Based on these values, the initial value of $103 \mathrm{ts}$ was considered, as well as 91 fixed increments of $6 t s$ to the final value of $648 t s$. Thus, 92 intermediary rates were considered between the minimum and maximum rates, which corresponds to a pool of 92 leaky buckets, each of which with a different $\mu i$ service rate, all submitted to the flow of sample-1.

Simulation was used to obtain, on the $\mathrm{j}$-th timeslot, for each leaky bucket, $\mathrm{Sj}$ values, cell in service (or not), and Qj, number of queued cells waiting to be attended. The estimated values of parameters $a, q$, and $b$ were then calculated through expressions (10) and (11). The other describing parameters of sample-1 were calculated next, from this set of basic values. Figures 6, 7 and 8 respectively present the graphs of parameters $a$, $b$ and $q$, obtained from the simulation of a pool of 92 leaky bucket shapers, each one associated to one of the different $\mu i$ service rates considered $(i=1,2, \ldots, 92)$, and submitted to the flow of sample-1. 


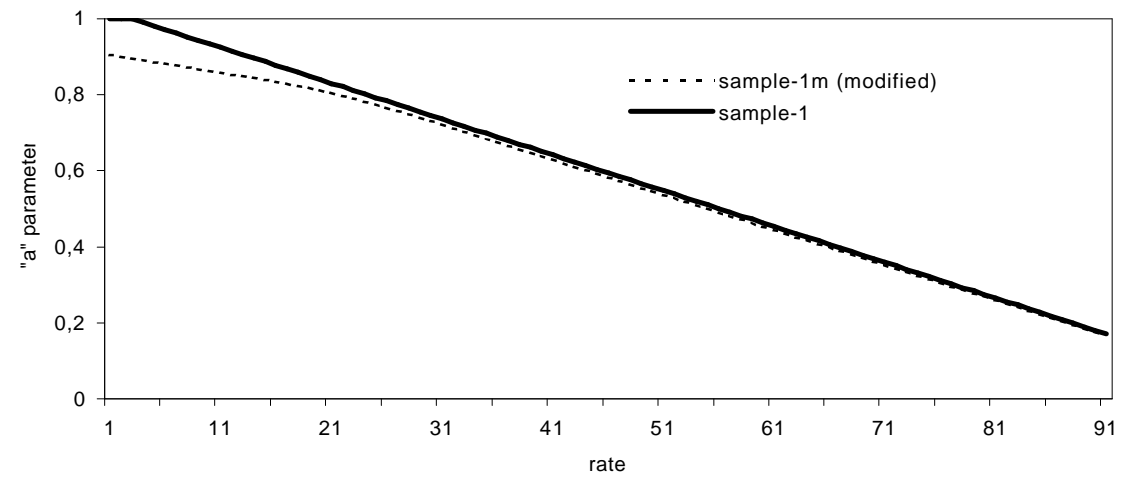

Fig. 6. Variation of parameter $a$ for sample-1 and sample-1m.

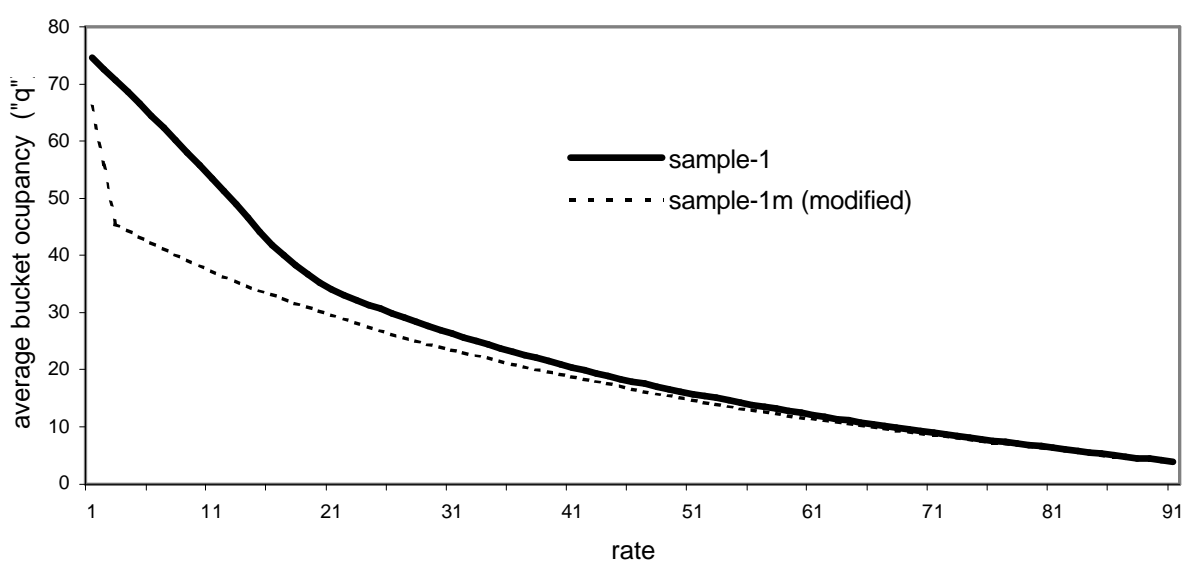

Fig. 7. Variation of parameter $q$ for sample-1 and sample-1m.

It can be noticed on figure 6 that the average value of parameter $a$, corresponding to the average value of cells in service on the leaky bucket for a given cell, decreases as the rate increases. Likewise, the average number of $q$ queued cells waiting to be attended, for a given rate, represents a significant increase as the service rate at the bucket exit decreases (Figure 7). As for parameter $b$ (Figure 8), associated to the inverse of the average delay that cells undergo in the shaper, it increases as the rate increases, i.e., delay decreases significantly with a rate increase.

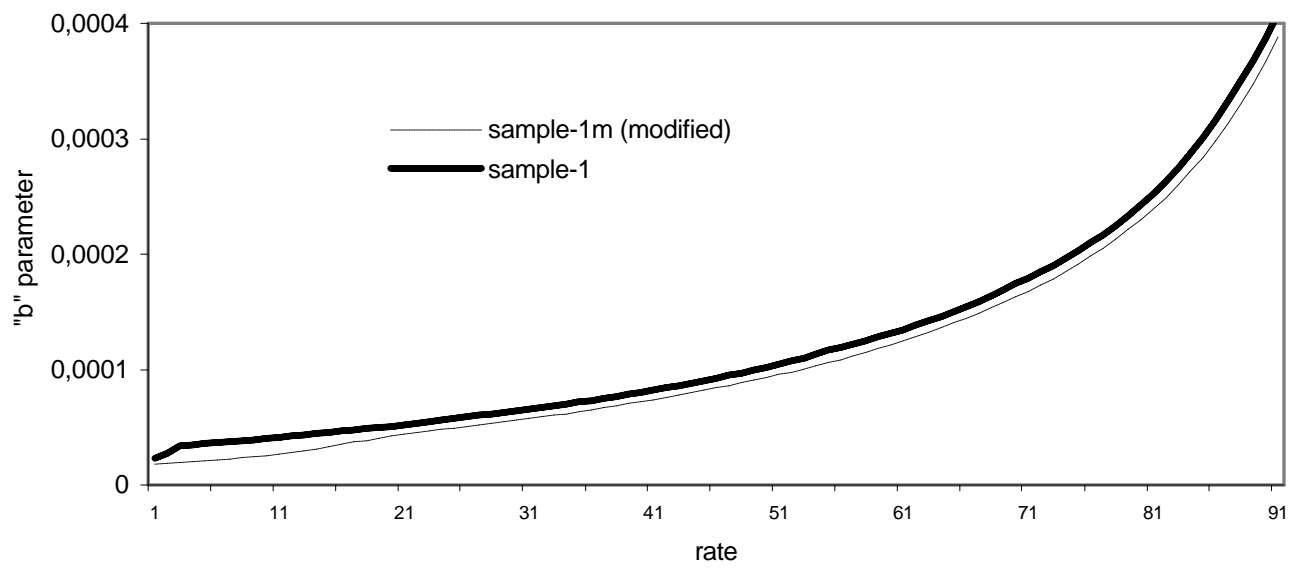

Fig. 8. Variation of parameter $b$ for sample-1. 
In order to show that parameters $a$ and $q$ capture flow variability, which can be measured by $C o V^{2}$ (coefficient of variation) and standard deviation $\sigma$ of sample-1, the method was applied to a modified flow of sample-1 called sample-1m. This modification consisted of considering, for all values of frames $\mathrm{I}, \mathrm{P}$ and $\mathrm{B}$ of sample-1, their mean values, whereas keeping the same limits for the considered rates. Therefore, a sample was obtained with the same burstness factor $(F r)$, but lower $\sigma$ and $C o V$ in relation to sample-1. It can be noted on Figure 6 that sample-1m features a greater $a$ average value, whereas $q$ is smaller, especially the lower rates. The behavior of both curves can be explained by the smaller variability of sample-1m, translated as smaller $\sigma$ and $\mathrm{CoV}$ values.

The graph on Figure 9 represents the minimum bucket size as a function of three different conformance probabilities. One can notice that the bucket size is greater with reduced rates for a given conformance probability. Likewise, the smaller the probability of conformance, the greater the requirements for buffer size.

Figure 10 presents the estimated capacity of the bucket as a function of the limit of average delay (D). A conforming probability $\varepsilon=0,001$ and a bucket capacity $B i$ were considered. It is noticeable that the limit is characterized by and decreases rapidly with an increased rate.

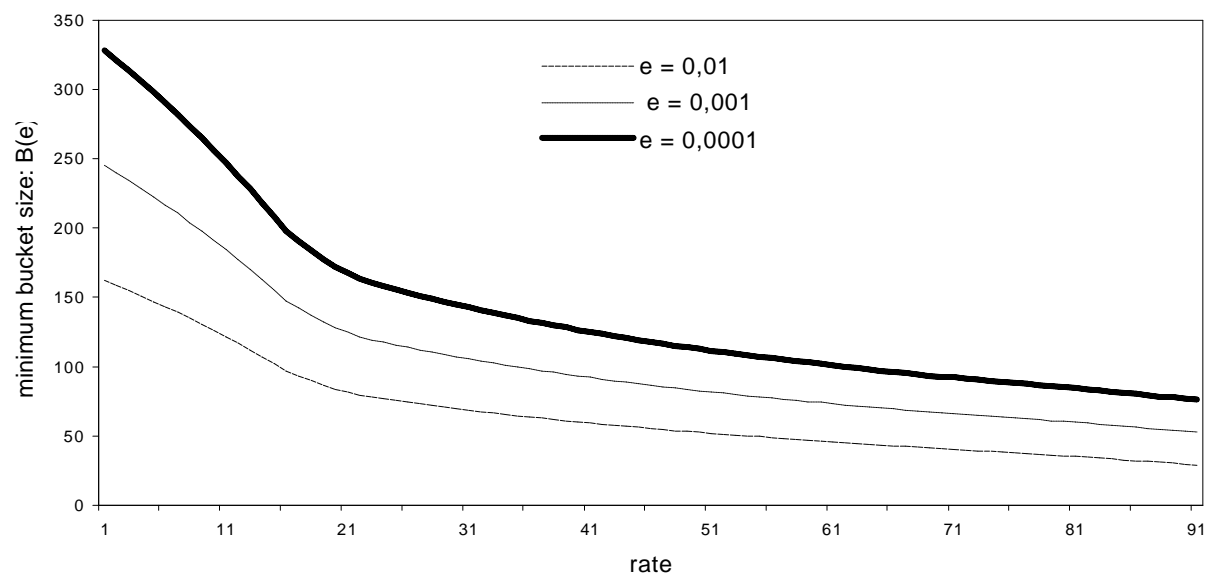

Fig. 9. Minimum bucket size (B) as a function of conforming probability $\varepsilon$.

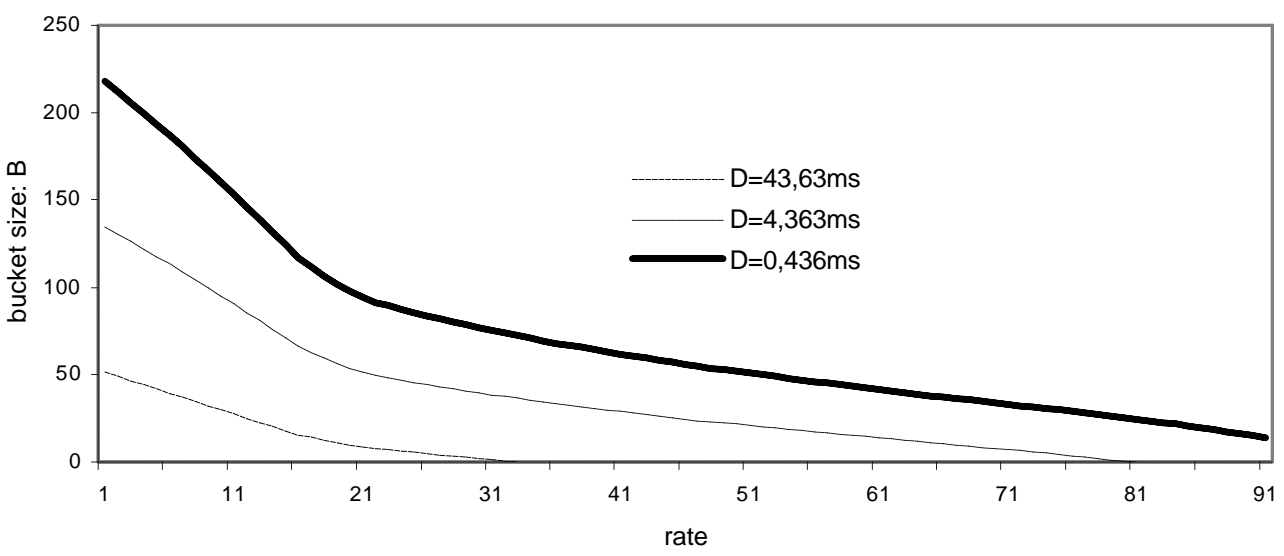

Fig.10. Bucket size for a given limit of average delay (D).

\footnotetext{
${ }^{2}$ Coefficient of Variation is defined as: $\mathrm{CoV}=\sigma \mathrm{X} / \mathrm{E}[\mathrm{X}]$
} 


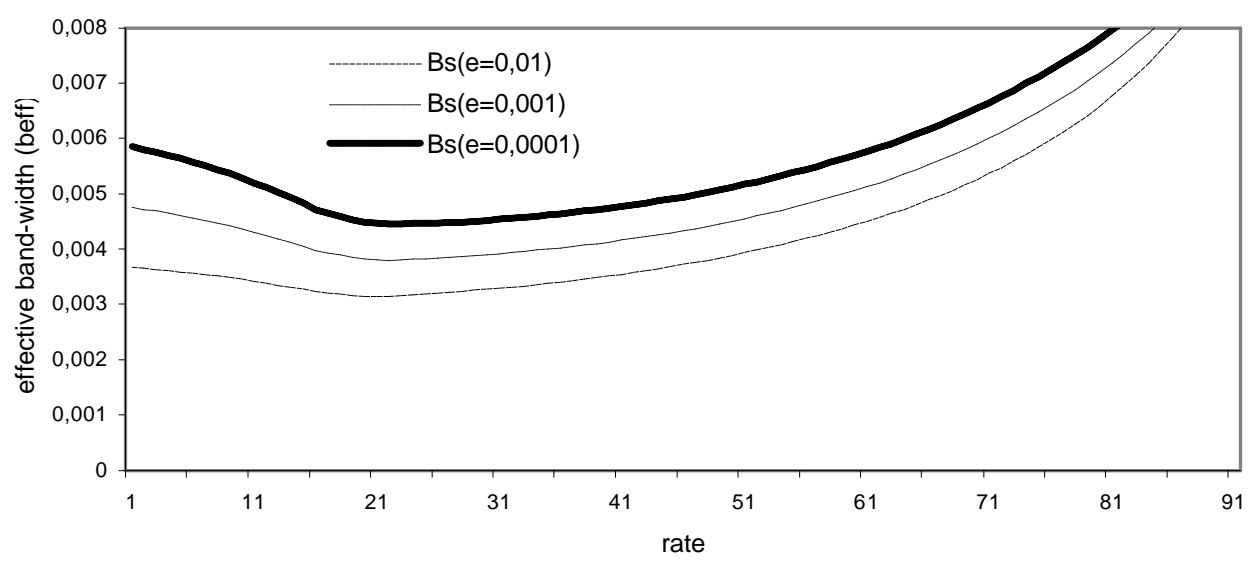

Fig. 11. Variation of effective band (beff) for different conforming probabilities.

Figure 11 presents the effective band associated to each rate considered. A factor $\mathrm{K}=0.01$ was adopted, based on [7], a typical Bmux of 400, a loss probability Emux of $10^{\wedge}-6$ and $k=C o V$ of 0,6 . The curve has an inflection point, corresponding to a minimum effective band of $3,776 \times 10^{\wedge}-3 \mathrm{cel} / \mathrm{ts}$ which, on its turn, corresponds to a minimum bucket capacity $\mathrm{Bs}=121$ cells (for a conforming probability $\varepsilon=0,001$ ), and a statistical rate $\lambda s$ of $1,912 \times 10^{\wedge}-3 \mathrm{cel} / \mathrm{ts}$. The maximum burst length (BT) that can be transmitted at peak rate $\lambda p$, is 144 cells. The estimated values of UPC parameters for sample-1, with conforming probability $\varepsilon=0,001$, are then: $\lambda \mathrm{s}=1,912 \times 10^{\wedge}-3 \mathrm{cel} / \mathrm{ts}, \lambda \mathrm{p}=9,550 \times 10^{\wedge}-3 \mathrm{cel} / \mathrm{ts}, \mathrm{Bs}=121$ cells and $\mathrm{BT}=144$ cells.

The other consecutive samples of the Star Wars video were analyzed under identical procedures. Figure 12 shows a graph of the approximate variation of parameters $\lambda p$ and $\lambda s$, of the ten samples along the given 40-second Star Wars flow. It can be noticed that each parameter of the traffic descriptor approximately follow the flow variation, as can be seen on Figure 4.

Figure 13 presents the variation of the bucket capacity (Bs) and the burst tolerance obtained for the ten considered samples. Again, there is a clear notion of how these parameters are directly related to flow progress, by comparing Figures 12 and 4.

In order to validate the practical method to obtain UPC parameters, a Double Virtual Scaling Shaper (DVSS) was simulated. Next, flows of each sample were applied to the Shaper, configured with the respective UPC parameters obtained with the practical methodology.

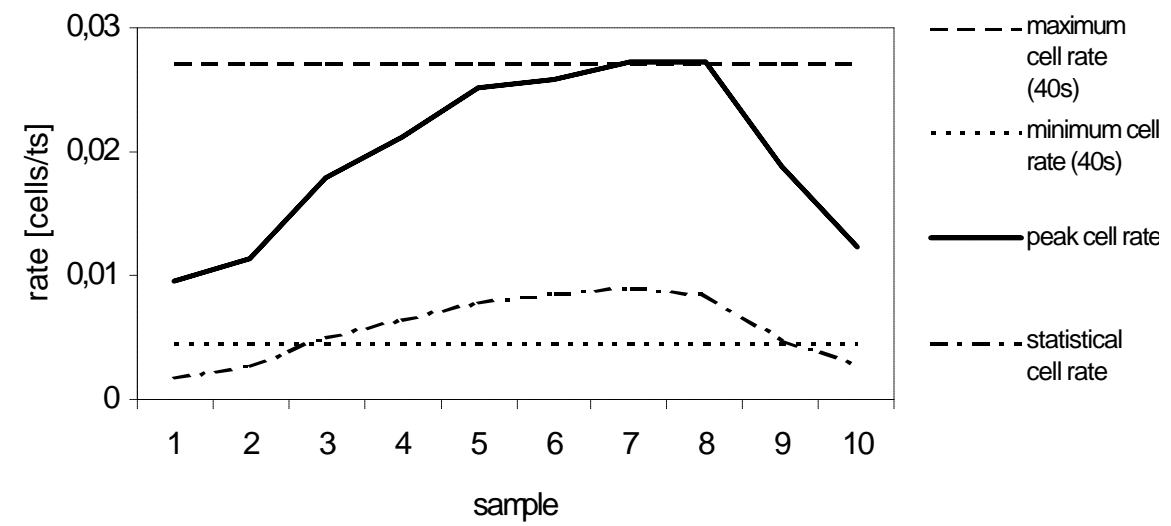

Fig. 12. Variation of peak rate $(\lambda \mathrm{p})$ and statistical rate $(\lambda \mathrm{s})$ in relation to the 40s-flow of MPEG video, Star Wars. 


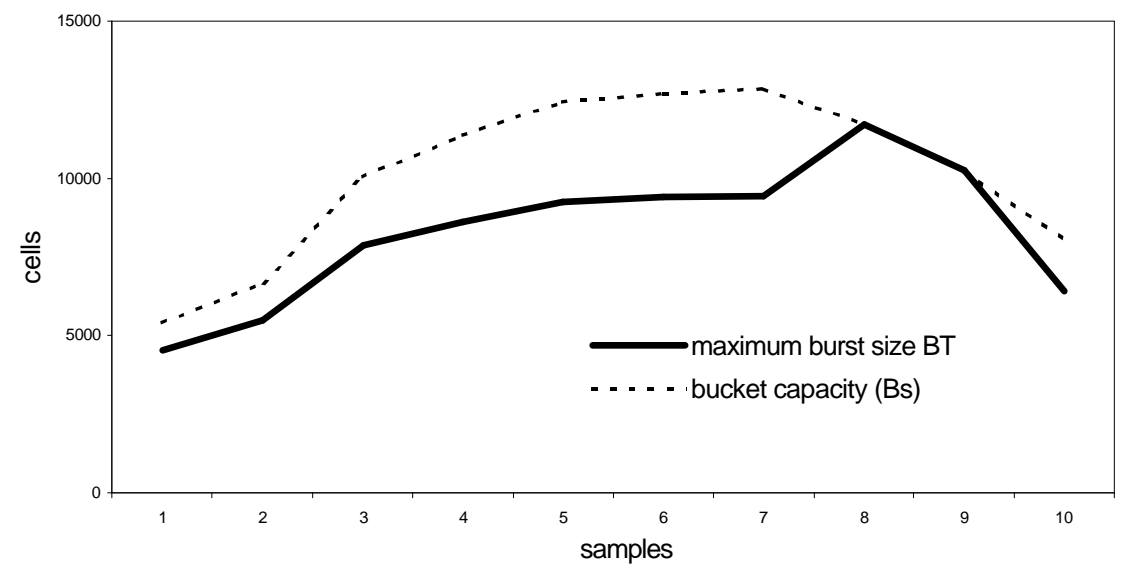

Fig. 13. Variation of bucket capacity (Bs) and burst tolerance (BT) observed throughout then ten samples considered.

The following parameters were observed in each simulation for each flow segment: the average and maximum numbers of cells on the shaper buffer and the number of buffer overflows (cell loss). From a total 65856 cells on the ten given samples, at no time did the buffer overflow.

It has been concluded that the practical method suggested for shaping real-time flows is valid and can be used to estimate approximate UPC parameters on any kind of VBR flow, within the sampling time interval $(\approx 4 \mathrm{~s})$ in which samples were considered. Thus, considering the dynamic renegotiation of UPC parameters of the virtual connection (VC) from successive segments of traffic flow, one can obtain a dynamic adaptation of the UPC mechanism and, therefore, the actual effective connection band, while it is on.

\section{CONCLUSIONS AND FUTURE DEVELOPMENTS}

Many ATM traffic flows either have no conditions to or are not concerned with supplying a reliable description of the statistical behavior of the flow they generate. It is fundamental that the application offer a precise and reliable traffic descriptor for the network to ensure application-required QoS.

This paper suggests a practical method to describe an ATM flow, based on an initial sampling of this flow, and the traffic parameters thus obtained can be renegotiated with the network whenever there is significant deviation along connection time. The model provides for flow shaping through a double leaky bucket shaper, which uses the UPC parameters obtained from the initial sample. Significant deviation of such parameters from current flow triggers a renegotiation with the network for more appropriate parameters, which makes the shaping process adaptive.

The adaptive real-time description and shaping of a traffic flow from the suggested practical method can be applied, in principle, to any service category. It must be noted, however, that this method will introduce a fixed delay that the application must tolerate. This delay is basically caused by three factors: processing time of the characterization algorithm, sampling time and renegotiation time of new UPC parameters with the network. Since these three processes, which characterize these time components, are run simultaneously, the fixed delay will be essentially defined by the longest procedure. An examination of each component, which follows, allows for an estimate of the delay to be introduced.

The algorithm runtime during simulation was around four seconds. This relatively long time is due to the fact that no special care was taken to program them in order to optimize processing time. We believe that special programming care will significantly reduce such time with little effort.

The flow sampling time conflicts with the algorithm processing time, because ideally the longer the sampling time, the more reliably the flow will be described, although the fixed delay will be the longer. We understand that these two time measurements are committed to each other for the definition of the fixed delay to be inserted in the flow.

The renegotiation of new UPC parameters with the network, on its turn, is run at the ATM signaling level, therefore there is no loss at the user data level. With new ATM signaling protocols, such as Q.2963.2 of ITU-T [1], the renegotiation time for a connection can be a few tens of milliseconds.

As for the way this renegotiation is carried out, it can be in two ways: regular renegotiation of UPC 
parameters from flow segments of a fixed size, or dynamic renegotiation - only run if there is a variation of UPC parameters below or above predefined limits. In both cases, the result will be an adaptive shaping of flow to an actual effective band that varies along time. The use of either kind of renegotiation (dynamic or periodical) must be decided with basis on characteristics such as flow variability and burstness.

As for the application of the methodology proposed, mostly its use of MPEG video flows was explored. Thus, video services such as stored video, video on demand $(\mathrm{VoD})$ and multicast video may benefit from the technology.

For better use of the potential behind this mechanism of adaptive optimization of the link, it is important to extend application of the algorithm to other kinds of sources, such as ON-OFF flows, assuming $\mathrm{ON}$ and OFF periods over random distributions. If we consider not too long sampling times ${ }^{3}$, the long term relations that are observed on most current network applications [9] can be satisfactorily modeled according to an exponential service rate distribution - besides a few predefined statistical parameters (service category, burstness, etc.) [13].

\section{REFERENCES}

[1] REININGER, D..; RAYCHAUDHURi, D.; OTT, M. A dynamic Quality of Service Framework for Video in Broadband Networks, IEEE Networks, November/December, v.12, n.6, p.22-34.

[2] ATM FORUM/TM. Traffic Management Specification Version 4.1, af-tm-0121.000, March 1999. Available on the Web. <URL: http://www.atmforum.com/atmforum/specs/approved.html >.

[3] DIAZ, J. C.; PLAZA, P.; CRESPO, J. ATM Traffic Shaper: ATS. In: DATE, 1998. Proceedings...[S. 1. : s. n.], 1998

[4] MARK, B. L.; RAMAMURTHY, G. Real-Time Estimation and Dynamic Renegotiation of UPC Parameters for Arbitrary Traffic Sources in ATM Networks. IEEE/ACM Transactions on Networking, New York, v. 6, n. 6, p. 811-827, Dec. 1998.

[5] MONTEIRO, J. A. S. Rede Digital de Serviços Integrados de Faixa Larga (RDSI-FL). Published by: Silvio Lemos Meira em, IX Escola de Computação, Recife, July, 206p, 1994.

[6] SIDI, M. et al. Congestion Control Through Input Rate Regulation, IEEE Transactions on Communications, New York, v. 41, n. 3, p.471-477, Mar. 1993.

[7] StAllingS, W. A Practical Guide to Queuing Analysis. BYTE, Peterborough, N. H., p. 309 - 316 , Feb. 1991.

[8] HEYMAN, D. P.; LAKSHMAN, T. V. Source Models for VBR Broadcast-Video Traffic. IEEE/ACM Transactions on Networking, New York, v. 4, n. 1, p. 40-48, Feb. 1996,

[9] ECKBERG, A. E. Approximations for bursty and smooted arrival delays based on generalized peakedness. In: INT. TELETRAFFIC CONGRESS, 11., 1985. Proceedings... Kyoto: [s. n.], 1985. p.234240.

[10] ROCHOL, J. Caracterização e Conformação de Fluxos de Tráfego ATM no Ambiente do Usuário, Doctor's disseration, Programa de Pós-Graduação do Instituto de Informática da UFRGS, December 2000, p.200.

[11] KNIGHTLY, E.; ZHANG, H. Traffic Characterization and Switch Utilization using a Deterministic Bounding Interval Dependent Traffic Model. Berkley: ICSI and EECS Department, U. C., 1994. 12p. (Technical Report TR-94-047) Available at: <ftp://ftp.icsi.berkeley.edu/pub/techreports/1994/tr-94047.ps.gz>. As accessed on: 17 October 2000.

[12] GALL, D. L. MPEG: A video compression standard for multimedia applications. Communications of the ACM, New York, v. 4, n. 34, p. 46-48, Apr. 1991.

[13] ABATE, J. et al. Asymptotics for steady-state tail probabilities in structured Markov queuing models. Stochastic Models, v. 10, p.99-134, 1994.

\footnotetext{
${ }^{3}$ The Time ( $\mathrm{t}$ ) was considered is the time of the average connection duration $(\mathrm{Tm})$. The condition $\mathrm{t}<<\mathrm{Tm}$ must be satisfied.
} 